\title{
Gemini Fiber for Interferometry and Sensing Applications
}

\author{
E. Zetterlund, A. Loriette, C. Sterner, M. Eriksson, H. Eriksson-Quist, and W. Margulis
}

Department of Fiber Photonics, Acreo, Electrum 236, 16440 Stockholm, Sweden

Correspondence should be addressed to W. Margulis, walter.margulis@acreo.se

Received 6 April 2009; Accepted 11 May 2009

Recommended by Valerio Pruneri

\begin{abstract}
A novel type of fiber for sensing applications is introduced based on a plurality of individual fiber preforms drawn together but maintaining their original shape. The individual cores of the Gemini fiber can be addressed at input and output ends through splicing to stand-alone fibers. Thermal coupling between cores is sufficient to eliminate relative thermal effects. Fabrication, coupling and fiber Bragg grating (FBG) studies are discussed. The possibility to connect in a single splice more than one fiber to the Gemini fiber is introduced as being highly advantageous. A conventional splicer can be used, the loss is acceptable and the operation takes minutes. Sensing with Gemini fibers is discussed mainly with the incorporation of fiber Bragg gratings. Another advantage of the concept of the Gemini fiber discussed is the possibility to make monolithic fiber interferometers of nearly equal arm lengths. A large number of new options are opened by the monolithic multicore fiber design, where access to individual cores is straightforward.
\end{abstract}

Copyright (c) 2009 E. Zetterlund et al. This is an open access article distributed under the Creative Commons Attribution License, which permits unrestricted use, distribution, and reproduction in any medium, provided the original work is properly cited.

\section{Introduction}

Multicore optical fibers [1] find a great number of applications in sensing beyond endoscopy. Various measurands have been monitored with the information extracted from the propagation of light in different cores [2-7]. The close proximity of the waveguides allows for equalizing thermal perturbations, and the temperature dependence is nearly eliminated by working with the difference between signals traveling in neighboring cores. Multicore fibers used in bend and torsion sensing can be employed in the determination of the path taken by a moving fiber incorporating a number of fiber Bragg gratings (FBGs), the reflected wavelengths indicating the bend experienced by the fiber along its path [8]. The input and output access to the individual cores, however, pose a severe coupling limitation. It has not been possible, so far, to splice individual fibers to the each core and guarantee low loss coupling. Various ingenious mounting schemes have been devised to make optical coupling possible [9-11], but they are generally lossy and costly, not particularly robust and ultimately far from ideal. Coupling at the input and output ends is so problematic that it significantly restricts the use of multicore fibers. In other applications, such as in telecom and pumping fiber lasers, independently drawn fibers are brought together in a ribbon or with low index coating materials [12]. Here, accessing the individual cores is straightforward, but the thermal and mechanical contact between fibers that would be useful for sensing is limited.

In the present letter, we describe a novel type of fiber for sensing applications. A single fiber is drawn from a number of individual fiber preforms and except for a narrow connecting glass bridge, the original individual circular cross-sections are maintained. A Gemini fiber is then obtained that has Siamese character. Addressing the individual cores at input and output ends thus becomes easier, through splicing to stand-alone fibers. Although thermal coupling is weaker than in a conventional multicore fiber, the easier access to the cores is greatly advantageous, and the structure is a good compromise for various sensing applications. Fabrication, coupling and fiber Bragg grating (FBG) studies are discussed in the following. Other advantages of this concept, including the possibility to make monolithic fiber interferometers of nearly equal arm lengths are also touched upon. The general name given for this type of structure is the Gemini fiber, and complementing information (e.g., G4) indicates the number of cores available. 


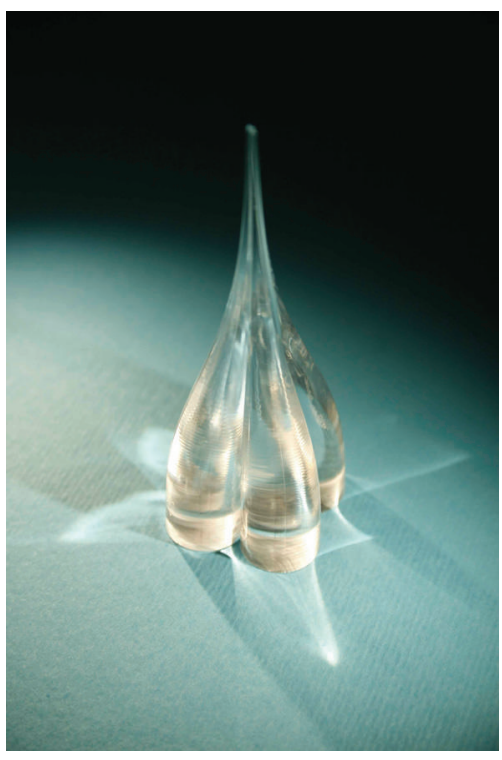

Figure 1: Preform of Gemini fiber G4, where four individual standard telecom fiber preforms are mounted together and drawn into a monolithic fiber with four easily accessed ports.

\section{Fiber Fabrication}

The fabrication of the Gemini fiber starts from the fixation of a number of close proximity preforms, which can have standard or special cross-section. Figure 1 shows one such arrangement, in which four standard telecommunications single-mode preforms are stacked. After drawing each preform results in an individual single mode fiber barely in contact with the neighboring fibers, as shown in Figure 1. Drawing is carried out without pressure control, but the furnace temperature $\left(\sim 1900^{\circ} \mathrm{C}\right)$ is slightly lower than in standard fiber manufacture. The automatic diameter control unit normally used while drawing is not used for feedback. This is necessary, since the width of the Gemini fiber seen from various angles varies, and the viewing angle changes during fiber drawing. When necessary, the temperature of the furnace is adjusted to prevent the dimensions of the fiber from drifting.

Four Gemini fiber types are illustrated in Figures 2(a)2(d) with scanning electron microscope pictures. These are (a) a Gemini fiber (G2), (b) a two-core Gemini fiber with holes $(\mathrm{G} 2 \mathrm{H}),(\mathrm{c})$ a Gemini fiber composed from four individual preforms (G4) shown in Figure 1, and (d) a Gemini fiber composed of four individual preforms and a middle rod $(\mathrm{G} 4 \mathrm{M})$. All individual preforms have circular shape and circular cores (except for the middle rod in Figure 2(d) that is coreless). Some form alteration was observed while drawing, in particular for the first Gemini fiber pulled G2 (Figure 2(a)). The measured distance between core centers in the G2 fiber is $135 \mu \mathrm{m}$ instead of the expected $125 \mu \mathrm{m}$ ( $8 \%$ distortion). This can be attributed almost entirely to the creation during drawing of the glass bridge connecting the individual halves of the fiber. The four individual diameters measured in G4 by fitting circles to the cross-section in
Figure 2(d) are $71.8 \mu \mathrm{m}, 72.7 \mu \mathrm{m}, 72.5 \mu \mathrm{m}$, and $72.0 \mu \mathrm{m}$. The distance between opposite core centers in two directions is $102.7 \mu \mathrm{m}$ and $101.5 \mu \mathrm{m}$. The four individual diameters measured in G4M by fitting circles to the cross section in Figure $2(\mathrm{~d})$ are $81.4 \mu \mathrm{m}, 80.0 \mu \mathrm{m}, 82.6 \mu \mathrm{m}$, and $81.0 \mu \mathrm{m}$.

\section{Accessing Individual Cores}

Two approaches are used for accessing the individual cores of the Gemini fibers. Here, work carried out with the Gemini fiber G2 is described in more detail. Etching is the first approach. From the cross-section seen in Figure 2(a) it is clear that the thin bridge connecting the individual waveguides is etched away relatively early if the fiber is immersed in HF. Two independent fibers result from this process, which can be spliced to standard fibers at both ends for input and output coupling. The time taken for separating two independent fibers was 18 minutes in a $40 \%$ concentration HF solution. A photo illustrating an etched Gemini fiber G2 is shown in Figure 3.

It is advantageous to strengthen the separation point, and UV curing glue (Wellomer UV 2010) is appropriate for this purpose. The two fiber sections can then be mounted, cleaved and spliced individually. It is found, however, that the cross-section of the two independent fibers resulting from etching the G2 is not circular, but maintains an edged profile. Even if the etching time is increased to 50 minutes, the two independent fiber sections are not circular. Three splicing options become available, those of automatically aligning the fibers to be spliced by imaging the claddings, the cores, or alternatively perform a manual splice. When the etched fiber sections are automatically aligned to the standard fiber from the cladding profile, a large mean loss of $1.7 \mathrm{~dB}$ is measured for the splice with a large standard deviation $(2.1 \mathrm{~dB})$. The lowest value obtained for the loss using this splice procedure is $0.36 \mathrm{~dB}$. Figure 4 illustrates one example of such a splice.

When the cores are automatically aligned, the minimum loss obtained improves to $0.14 \mathrm{~dB}$ with a standard deviation of $0.87 \mathrm{~dB}$, but splicing was only successful in approximately half of the cases. Finally, when a manual aligning procedure is used, the mean loss is $0.53 \mathrm{~dB}$, the standard deviation $0.56 \mathrm{~dB}$ and the minimal loss measured is as low as $0.05 \mathrm{~dB}$. All splices carried out manually were successful. One disadvantage of splicing individually the separated fiber sections to standard fibers is the long length of unprotected fiber created. Besides, whenever a fiber section breaks or a splice is unsuccessful, at least one additional fiber needs to be recleaved to approximately equalize the lengths, or in the worst case start the etching procedure again. As a whole, the etching procedure described for separating the individual waveguides gives acceptable results. However, it involves many steps, including coating removal, etching, fixing, cleaving, manual splicing four times and final mounting. The total time estimated for these operations carried out manually is at present in excess of three hours.

The second approach developed for accessing the individual cores of the Gemini fiber to independent standard telecom fibers is to perform a single automated fusion splice 


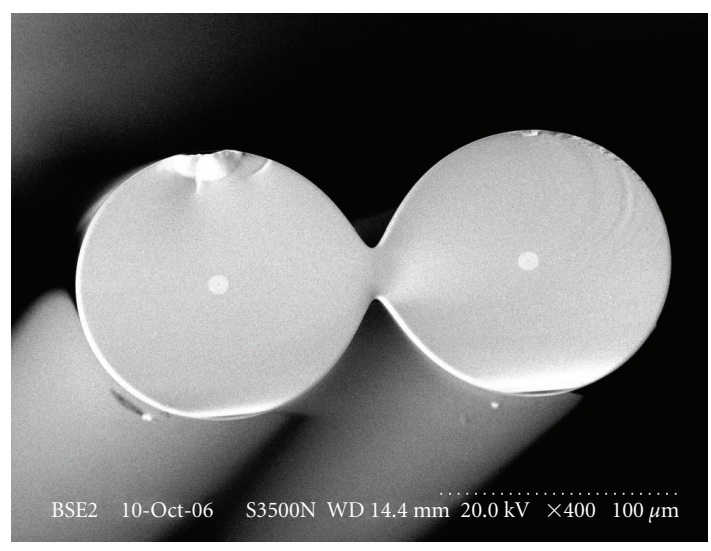

(a)

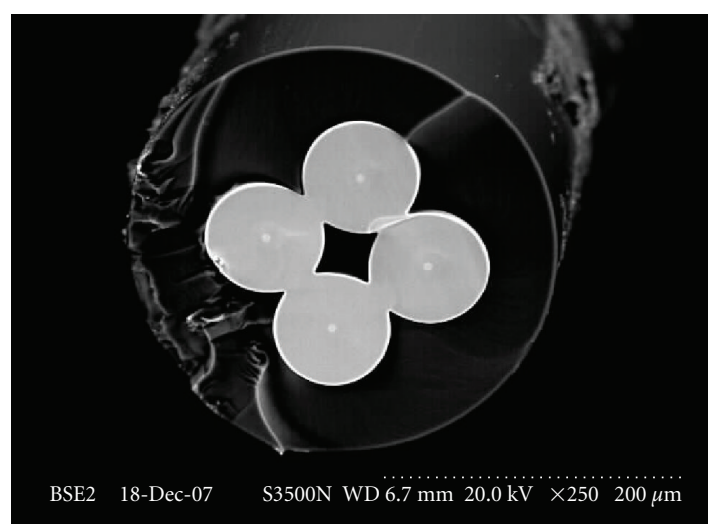

(c)

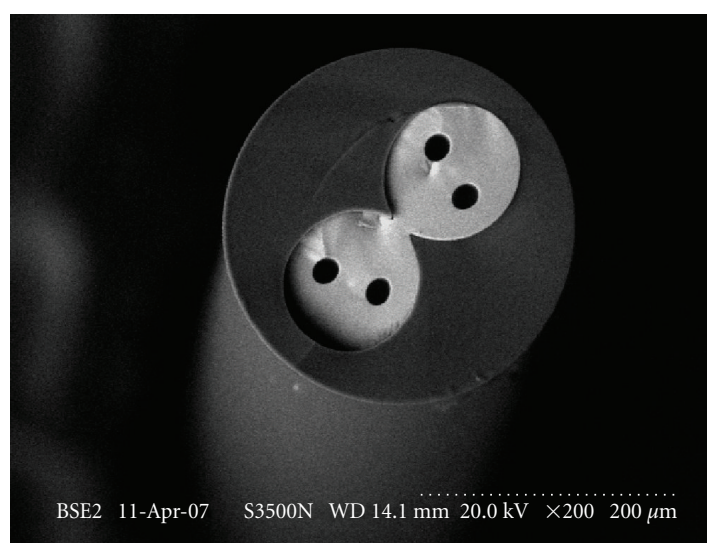

(b)

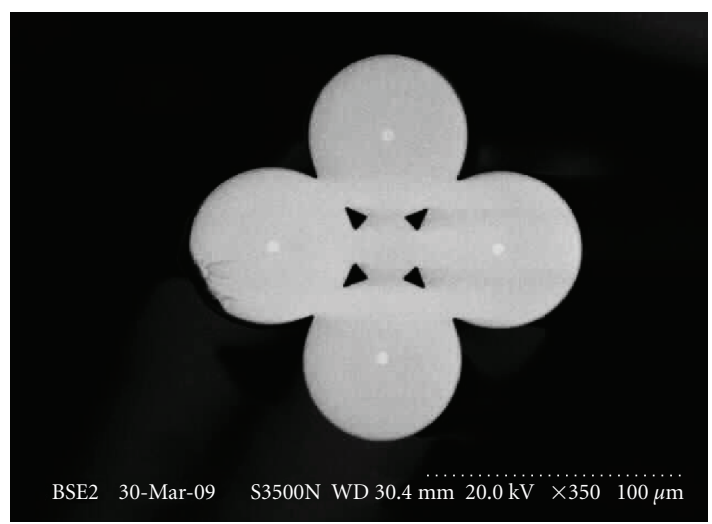

(d)

FIgURE 2: SEM pictures of Gemini fibers. (a) Gemini fiber G2; (b) Gemini fiber with holes (G2H); (c) Fiber G4; and (d) Gemini fiber G4M with a glass rod in the middle.

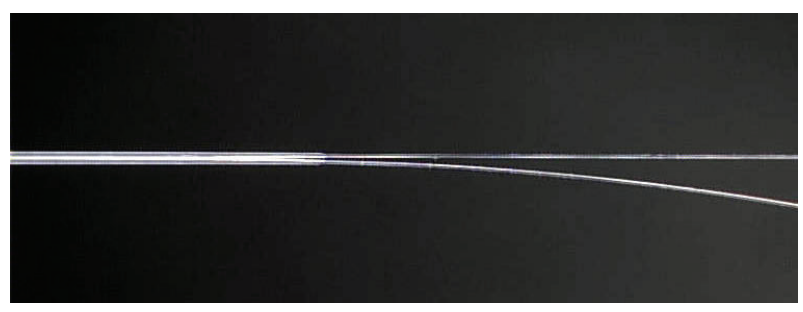

Figure 3: Gemini fiber G2 where etching is used to separate the arms for splicing to standard telecom fiber. After separation, the splitting point is reinforced with UV curing glue.

of various fibers in parallel. This procedure is also studied in some detail for the Gemini fiber G2. With this procedure, one exploits the ability to cleave well the Gemini fiber with a conventional cleaver, producing a fiber with various waveguides of equal length (as seen in Figure 2). Here, the entire process of addressing the individual cores is reduced to a couple of splices. The handling time is dramatically reduced. The resulting component becomes rugged with the use of conventional splice protectors. The main disadvantage of a single splice to each side of the Gemini fiber is that no

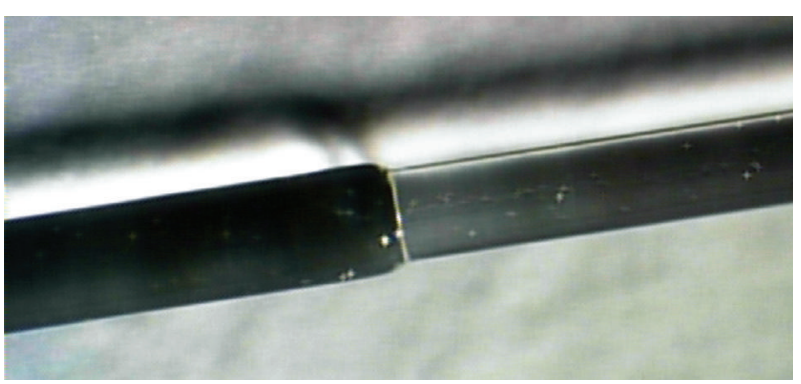

FIGURE 4: One of the two waveguides of the Gemini fiber G2 spliced to a standard telecom fiber through automatic alignment between cladding images.

individual fiber adjustment becomes possible. While this is easier when the core separation is exactly $125 \mu \mathrm{m}$ because the individual fibers are placed in contact with each other, mounting individual standard telecom fibers in parallel separated by a few microns requires a special fixture. For splicing the Gemini fiber G2 available in this work where the core center separation is $135 \mu \mathrm{m}$, an appropriate fiber holder was fabricated with two grooves separated by $135 \mu \mathrm{m}$. The result gives typically a loss below $1 \mathrm{~dB}$ per splice, but a loss below $0.5 \mathrm{~dB}$ has also been achieved for each one of the 


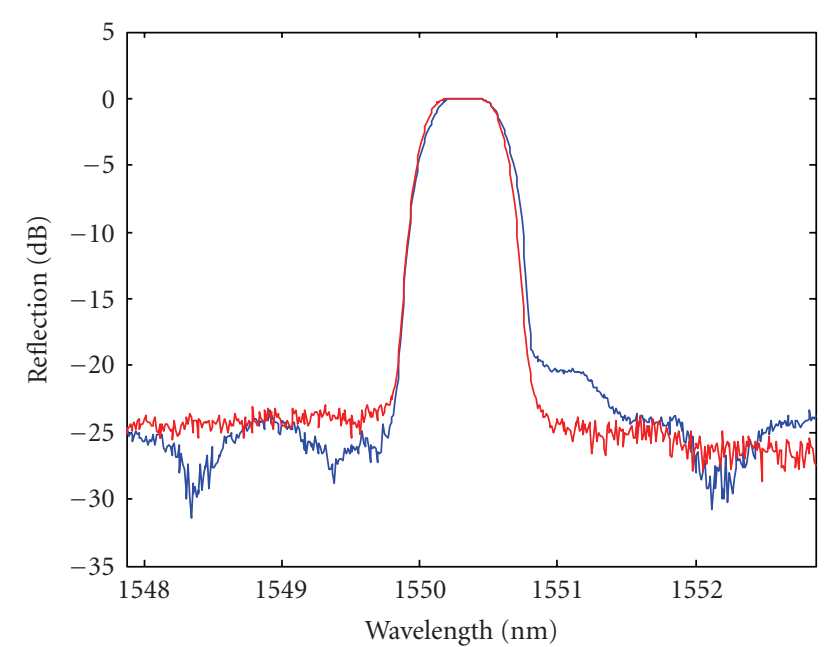

(a)

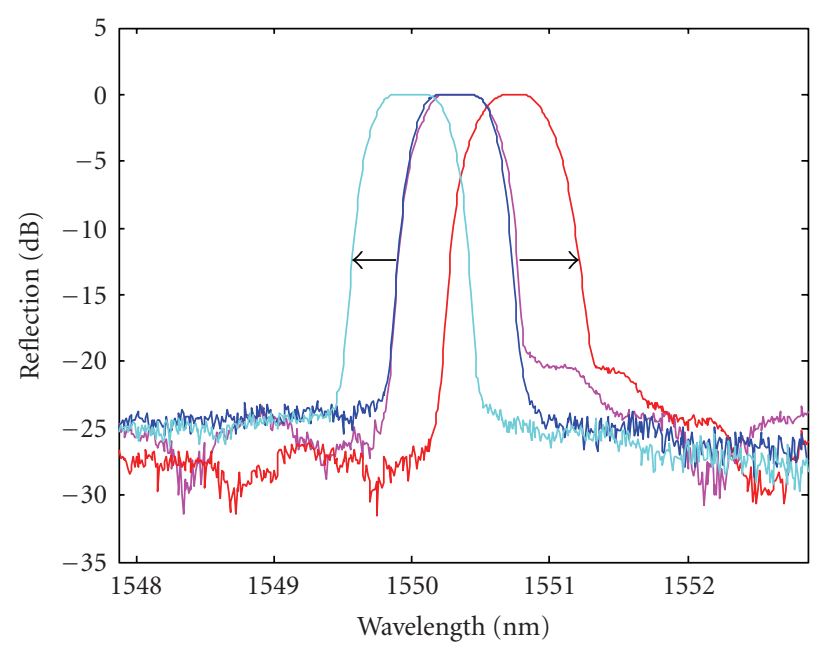

(b)

Figure 5: (a) Two fiber Bragg gratings written one in each core of a Gemini G2 fiber. (b) Upon bending, one grating shifts to shorter wavelengths while the other one shifts to longer wavelengths.

pair of fibers spliced to the Gemini G2 fiber. The total splice procedure to each Gemini fiber end here takes $\sim 15$ minutes if the loss is considered acceptable.

\section{Sensing with Bragg Gratings}

One of the most interesting applications of the Gemini fiber concept involves writing Bragg gratings in the various cores. Similarly to conventional multicore fibers, the sensitivity to temperature of the various waveguides is similar, and if the temperature varies all gratings shift equally in wavelength. However, a bend around the neutral axis cause the FBG to shift to longer wavelengths when the waveguide is elongated and to shorter wavelengths when it is compressed [13-15]. It is thus possible to determine optically the strain or stress to which the fiber is subjected. By translating other measurands such as pressure or acceleration to a bend, the Gemini fiber can be employed in various sensing applications. Here, we describe preliminary studies of Gemini fibers (G2) with fiber Bragg gratings.

The qualitative behavior of the Gemini G2 fiber is illustrated in Figure 5. A fiber Bragg grating is written in each one of the (in this case two) cores. The process is done sequentially, one grating at a time. The fiber is previously $\mathrm{H}_{2}$-loaded at $150 \mathrm{~atm}$ for two weeks at room temperature. UV exposure is carried out in an interferometer with $244 \mathrm{~nm}$ radiation from a frequency doubled $\mathrm{CW} \mathrm{Ar}^{+}$laser delivering $\sim 23 \mathrm{~mW}$. In the particular example here, the $1.2-\mathrm{cm}$ long unchirped Hamming apodized FBGs are both centered at $\sim 1550 \mathrm{~nm}$, are $\sim 0.5 \mathrm{~nm}$ broad and relatively strong $(\sim 25 \mathrm{~dB})$ after 7 identical exposures.

Upon bending around its own axis, one grating shifts to longer wavelengths and the other one to shorter wavelengths, as seen in Figure 5. In order to carry out a more quantitative measurement, another pair of gratings was recorded in a piece of Gemini fiber G2, which after recording was mounted

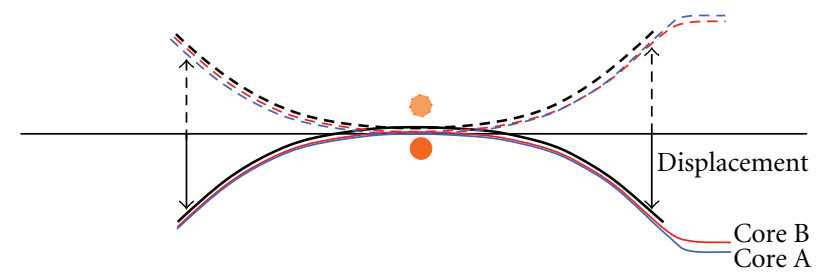

FIgure 6: Schematic diagram of FBG bending set-up. Solid drawings indicate compression and dashed stretching. The scheme is not in scale.

with UV curing glue on a flexible beam [13-15]. The piece of G2 fiber is displaced from the axis of the beam so as to result in a large wavelength shift when the beam is bent. The wavelengths of the two gratings are matched at $1555 \mathrm{~nm}$ at room temperature with the beam straight.

Figure 6 shows the schematic set-up used to study the response to a bend (displacement) of FBGs written in a Gemini G2 fiber. The bend is introduced by displacing the two extremes of the beam while keeping the center fixed as seen in Figure 6. A $5 \mathrm{~mm}$ displacement here corresponds to a bend radius of $25 \mathrm{~cm}$. Negative values mean that the gratings are elongated and positive displacements correspond to compression.

Figure 7 illustrates the tuning of the Bragg wavelengths for the two FBG of this device. The parabolic (black and red) curves shown are the best fit to the data (squares for core $\mathrm{A}$ and circles for core $\mathrm{B}$ ) of the Bragg wavelength when the beam is bent. A tuning range of $16 \mathrm{~nm}$ is covered with a displacement $\mathrm{D}$ in the range $-2 \mathrm{~mm}<D<+10 \mathrm{~mm}$, where $D$ is measured $5 \mathrm{~cm}$ from the center of the grating. Figure 7 also gives the difference in Bragg wavelengths for cores A and $\mathrm{B}$ measured when the bend is increased. A well-behaved and monotonic shift is observed (blue triangles), indicating that this difference can be used as an optical measurement 


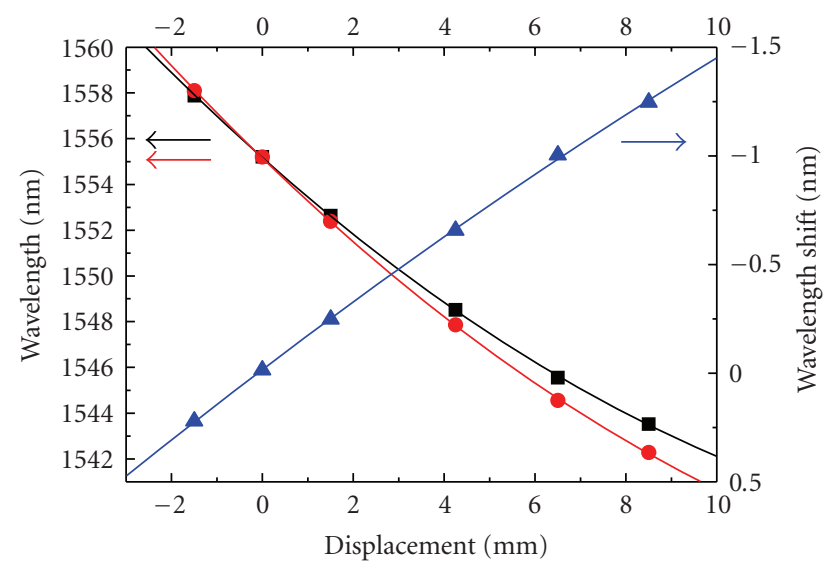

Figure 7: Bend tuning a Gemini S2 fiber with two FBGs. Both Bragg wavelengths shift by $>14 \mathrm{~nm}$. Triangles show the difference between the two waveguides. Measurements carried out at room temperature.

of the displacement. This device broke before temperature measurements could be carried out.

A third device was then manufactured in Gemini G2 fiber, where the two Bragg wavelengths do not overlap $\left(\lambda_{A}=\right.$ $1533.3 \mathrm{~nm}$ and $\lambda_{B}=1535.5 \mathrm{~nm}$ at $25^{\circ} \mathrm{C}$ ). This device was mounted as schematically shown in Figure 6 and heated with a lamp. The temperature of the arrangement was monitored with a thermocouple in close proximity to the FBGs. No particular precaution was taken to equalize the temperature at the waveguides, equalization relying only on the close proximity of the cores. Figure 8 (a) shows a plot of the Bragg wavelengths for both cores at $25^{\circ} \mathrm{C}, 35^{\circ} \mathrm{C}$ and $45^{\circ} \mathrm{C}$, where $D$ is measured $13.5 \mathrm{~cm}$ from the center of the grating. The solid curves are again parabolic fits to the data. In contrast to the behavior in Figure 7, here the second derivative of the solid lines is negative. The reason for this difference is not understood at present, but is likely to be associated to the exact position in relation to the axis of the beam at which the Gemini device is glued, which is largely uncertain and strongly affects the response of the gratings. It is clear from Figure 8(a) that the peak wavelengths change both with the bend (displacement) and with temperature. The sensitivity to temperature measured is $32 \mathrm{pm} /$ Kelvin. This is approximately 3 times larger than expected for a freestanding FBG, indicating that the plastic beam and glue dominate the temperature dependence. A similar problem is likely to be found in most applications where a Gemini fiber G2 is to be used for sensing. The relative wavelength shift, that is, the difference between the shifts for the two cores is determined for various bends at three temperatures. It is hoped that the temperature dependence can be cancelled out exactly, and only the bend (displacement) remains. Figure 8 (b) illustrates the three curves obtained at $25^{\circ} \mathrm{C}$, $35^{\circ} \mathrm{C}$ and $45^{\circ} \mathrm{C}$ from the subtraction of the pair of blue curves (triangles), red curves (circles) and black curves (squares). A set of three monotonic curves is obtained, relating the wavelength to the displacement. The curves nearly overlap, indicating that the temperature effects can be cancelled from the information from both cores. The maximum deviation measured amounts to $0.5 \%$. Thus, it is concluded that the bend can be determined from the difference in Bragg wavelengths for the two gratings. It should be remarked that the Gemini G2 fiber tends to bend naturally in such a way that both cores (FBGs) are affected equally much. In order to have one core stretching more than the other when bending, the fiber needs to be mounted on a beam and fixated (e.g., by gluing). The G4 fiber, on the other hand, can be easily bent in various directions and the fixation requirements are relaxed.

From these preliminary experiments it can be concluded that the Gemini fibers can be used with fiber Bragg gratings for sensing bends and displacement. Temperature effects can be removed using the information acquired from both cores.

\section{Interferometry with Gemini Fiber}

One interesting potential application of the Gemini fiber is in the embodiment of a monolithic fiber interferometer. The structure of the fibers fabricated in the drawing tower (cf. Figure 2) is well suited for the manufacture of fusion couplers. Thus, the easy access to each core, the possibility to taper a Gemini fiber locally to allow for coupling between cores and the rigid structure which guarantees equal arm lengths and minimizes polarization uncertainty provide for a useful broadband fiber interferometer. Potential applications include electrically driven fiber switches and modulators and add-drop multiplexers [16-18].

A preliminary experiment was carried out to make a coupler in Gemini G2 fiber. A Vytran LDS-1250 splicing/tapering machine with a 3-mm tungsten element is employed. The computer driven procedure consists first of a fusion stage, when the heated zone is scanned over a $10 \mathrm{~mm}$ section of the G2 fiber so that the waveguides start fusing together. This process takes 1500 seconds, and a fusion coefficient $[19,20] 0.45$ is obtained (i.e., the normalized ratio of the structure reducing from 2 to 1.73 ). The fusion stage is followed by tapering stage, when the fused zone is simultaneously heated and pulled to become thinner. The process takes 2000 seconds and generates an adiabatic structure. Because of the nonoptimal coupler fabrication process and in particular the metal oxide layer deposited on the taper, the excess loss incurred is $\sim 3.3 \mathrm{~dB}$. This is unacceptably high for a commercial device, but still usable for characterization of the Gemini fiber. The optical set-up used is for monitoring the process is illustrated in Figure 9.

The fabrication of the coupler in a Gemini fiber implies in the creation of a Michelson interferometer [21], where the back reflection is obtained from the fiber cleave. If the interferometer is probed with a white light source, the bandwidth obtained indicates how well the two optical paths match. Figure 10 illustrates the result of such a measurement. The extinction measured is $14 \mathrm{~dB}$. From the $50 \mathrm{~nm}$ FWHM bandwidth, the optical path difference inferred for the 40$\mathrm{cm}$ long device is $12.8 \mu \mathrm{m}$ (i.e., $32 \mu \mathrm{m} / \mathrm{m}$ ). It is possible that the path unbalance is created either at the coupler or at the end cleave. However, if the unbalance is assigned entirely to a difference in propagation constants between the two fiber 


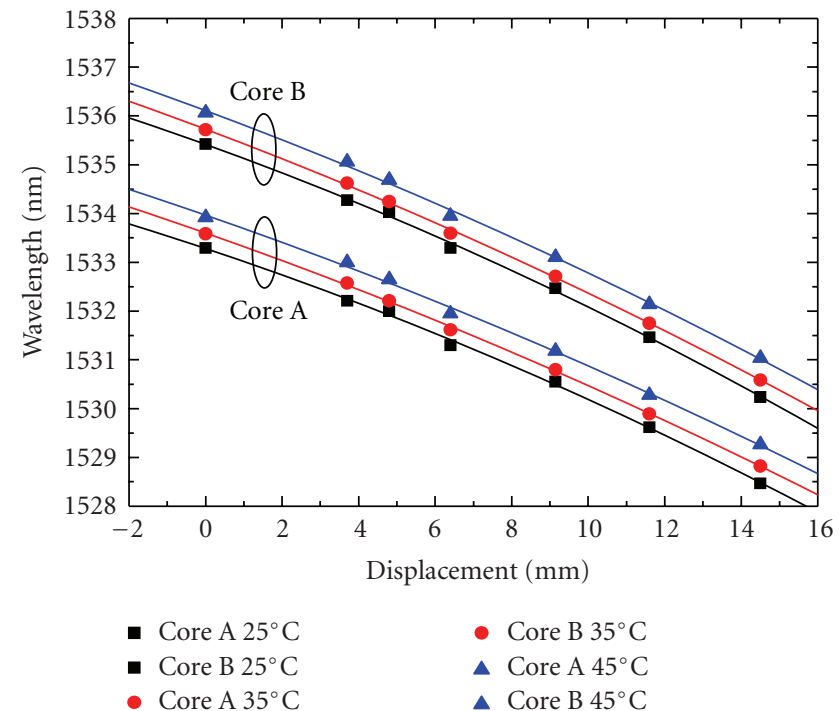

(a)

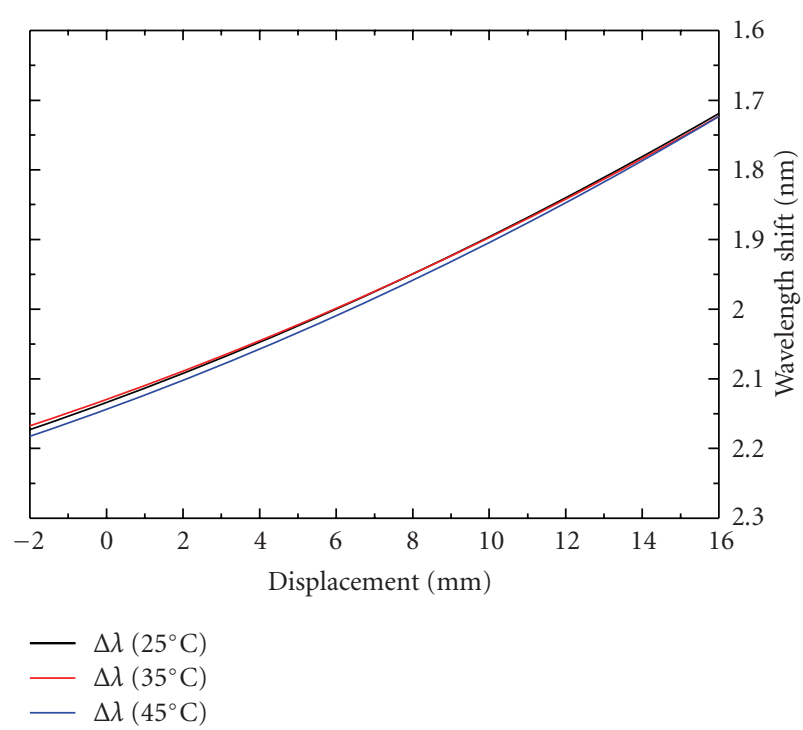

(b)

Figure 8: (a) Bragg wavelength $\lambda_{A}$ and $\lambda_{B}$ measured for cores A and B when a piece of Gemini fiber G2 with FBGs is compressed (positive values of the displacement) and stretched (negative values) at $25^{\circ} \mathrm{C}, 35^{\circ} \mathrm{C}$ and $45^{\circ} \mathrm{C}$. (b) Relative wavelength shift $\lambda_{B}-\lambda_{A}$ at three temperatures. The displacement is associated to wavelength and the temperature dependence is small.

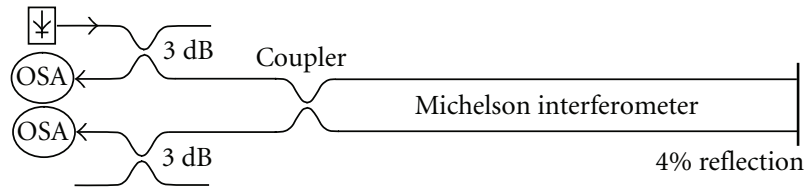

FIGURE 9: Arrangement used for the fabrication and characterization of fused fiber couplers and interferometers in Gemini G2 fiber.

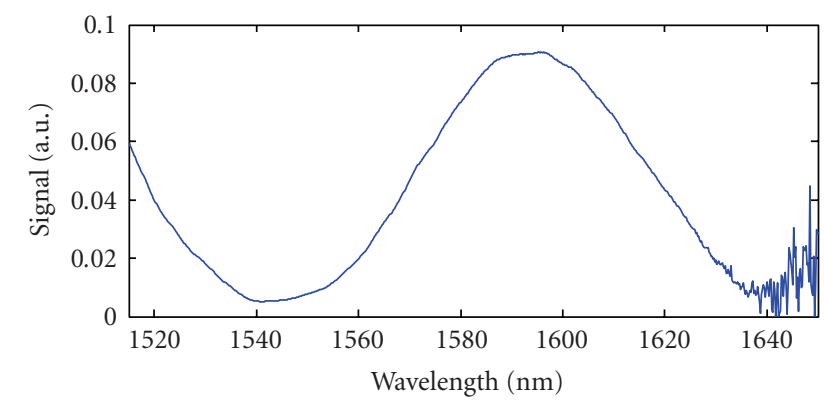

Figure 10: Measurement of the optical bandwidth of a $40 \mathrm{~cm}$ long fiber interferometer on a linear scale. The FWHM is $\sim 50 \mathrm{~nm}$.

cores, a mode index difference of $3 \times 10^{-5}$ is obtained. This is an upper limit for the inequality of the two waveguides in the Gemini fiber G2.

Other characterization measurements were carried out. The differential group delay (DGD) of $2 \mathrm{~m}$ long section of Gemini G2 and G2H fiber is measured to be $\sim 27 \mathrm{fs}$ and $\sim 74 \mathrm{fs}$, respectively, and the polarization dependent loss (PDL) of G2 for the same length is $0.1 \mathrm{~dB}$. These low values are perhaps not surprising considering that the fibers are made from standard telecommunication preforms.

\section{Conclusions}

A novel fiber configuration is introduced in this work, based on a plurality of individual fiber preforms drawn together but nearly maintaining their original shape. This concept can be extended to the case where the preforms are unequal, for instance, with one arm drawn from a single-mode and the other from a microstructured, doped or multimode preform. Coupling is studied in the present work and the possibility to connect in a single splice more than one fiber to the Gemini fiber is introduced as being highly advantageous. A conventional splicer can be used, the loss is acceptable and the operation takes minutes. Sensing with Gemini fibers is discussed mainly with the incorporation of fiber Bragg gratings. The temperature dependence of devices made from this fiber type can be removed almost entirely by using the information collected from both cores. Interferometry is also briefly discussed, although it can be appreciated that a large number of new options are opened by the monolithic multicore fiber design, where access to individual cores is straightforward.

\section{Acknowledgments}

It is a pleasure to acknowledge the valuable input of Suzanne Lacroix on the procedure to be used for the fabrication of fused couplers used in this work. Help by Oleksandr Tarasenko, Erik Petrini, Per Helander and Håkan Olsson at Acreo is also greatly appreciated.

\section{References}

[1] A. Méndez and T. S. Morse, Eds., Specialty Optical Fibers Handbook, chapter 6.4, Elsevier, Amsterdam, The Netherlands, 1st edition, 2007. 
[2] J. W. Arkwright, S. J. Hewlett, G. R. Atkins, and B. Wu, "Highisolation demultiplexing in bend-tuned twin-core fiber," Journal of Lightwave Technology, vol. 14, no. 7, pp. 1740-1745, 1996.

[3] M. J. Gander, E. A. C. Galliot, R. McBride, and J. D. C. Jones, "Bend measurement using multicore optical fiber," in Optical Fiber Sensors, OSA Technical Digest Series, Optical Society of America, 1997, OWC6.

[4] M. J. Gander, D. Macrae, E. A. C. Galliot, et al., "Twoaxis bend measurement using multicore optical fibre," Optics Communications, vol. 182, no. 1-3, pp. 115-121, 2000.

[5] G. M. H. Flockhart, W. N. MacPherson, J. S. Barton, J. D. C. Jones, L. Zhang, and I. Bennion, "Two-axis bend measurement with Bragg gratings in multicore optical fiber," Optics Letters, vol. 28, no. 6, pp. 387-389, 2003.

[6] G. M. H. Flockhart, G. A. Cranch, and C. K. Kirkendall, "Differential phase tracking applied to Bragg gratings in multicore fibre for high accuracy curvature measurement," Electronics Letters, vol. 42, no. 7, pp. 17-18, 2006.

[7] M. Silva-Lopez, C. Li, W. N. MacPherson, et al., "Differential birefringence in Bragg gratings in multicore fiber under transverse stress," Optics Letters, vol. 29, no. 19, pp. 2225-2227, 2004.

[8] C. G. Askins, T. F. Taunay, G. A. Miller, et al., "Inscription of fiber Bragg gratings in multicore fiber," in Bragg Gratings, Photosensitivity and Poling in Glass Waveguides (BGPP '07), p. JWA39, Quebec, Canada, 2007.

[9] S. B. Poole and J. D. Love, "Single-core fibre to twin-core fibre connector," Electronics Letters, vol. 27, pp. 1559-1560, 1991.

[10] R. R. Thomson, H. T. Bookey, N. D. Psaila, et al., "Ultrafastlaser inscription of a three dimensional fan-out device for multicore fiber coupling applications," Optics Express, vol. 15, no. 18, pp. 11691-11697, 2007.

[11] L. Yuan, Z. Liu, J. Yang, and C. Guan, "Bitapered fiber coupling characteristics between single-mode single-core fiber and single-mode multicore fiber," Applied Optics, vol. 47, no. 18, pp. 3307-3312, 2008.

[12] A. B. Grudinin, D. N. Payne, P. W. Turner, et al., "Multi-fiber arrangements for high power fiber lasers and amplifiers," US patent 6826335, November 2004.

[13] T. Imai, T. Komukai, and M. Nakazawa, "Dispersion tuning of a linearly chirped fiber Bragg grating without a center wavelength shift by applying a strain gradient," IEEE Photonics Technology Letters, vol. 10, no. 6, pp. 845-847, 1998.

[14] S. Y. Set, B. Dabarsyah, C. S. Goh, et al., "A widely tunable fiber Bragg grating with a wavelength tunability over $40 \mathrm{~nm}$," in Proceedings of the Optical Fiber Communication Conference and Exhibit (OFC '01), vol. 1, pp. MC4-1-MC4-3, 2001.

[15] C. S. Goh, M. R. Mokhtar, S. A. Butler, S. Y. Set, K. Kikushi, and M. Ibsen, "Wavelength tuning of fiber Bragg grating over $907 \mathrm{~nm}$ using a simple tuning package," IEEE Photonics Technology Letters, vol. 15, no. 4, pp. 557-559, 2003.

[16] R. Kashyap, Fiber Bragg gratings, chapter 6.4, Academic Press, New York, NY, USA, 1st edition, 1999.

[17] P. Yvernault, D. Mechin, E. Goyat, L. Brilland, and D. Pureur, "Fully functional optical add \& drop multiplexer using twin-core fiber based Mach-Zehnder interferometer with photoimprinted fiber Bragg gratings," in Proceedings of the Optical Fiber Communication Conference and Exhibit (OFC'01), vol. 3, pp. WDD92-1-WDD92-3, 2001.

[18] W. Margulis, O. Tarasenko, and N. Myrén, "Electrooptical fibers," in Photonics North, P. Mathieu, Ed., vol. 6343 of Proceedings of SPIE, Quebec, Canada, 2006, 634319.
[19] E. Pone, X. Daxhelet, and S. Lacroix, "Refractive index profile of fused-tapered fiber couplers," Optics Express, vol. 12, no. 13, pp. 2909-2918, 2004.

[20] E. Pone, X. Daxhelet, and S. Lacroix, "Refractive index profile of fused-fiber couplers cross-section," Optics Express, vol. 12, no. 6, pp. 1036-1044, 2004.

[21] R. Kashyap and B. K. Nayar, "An all single-mode fiber Michelson interferometer sensor," Journal of Lightwave Technology, vol. 1, no. 4, pp. 619-624, 1983. 

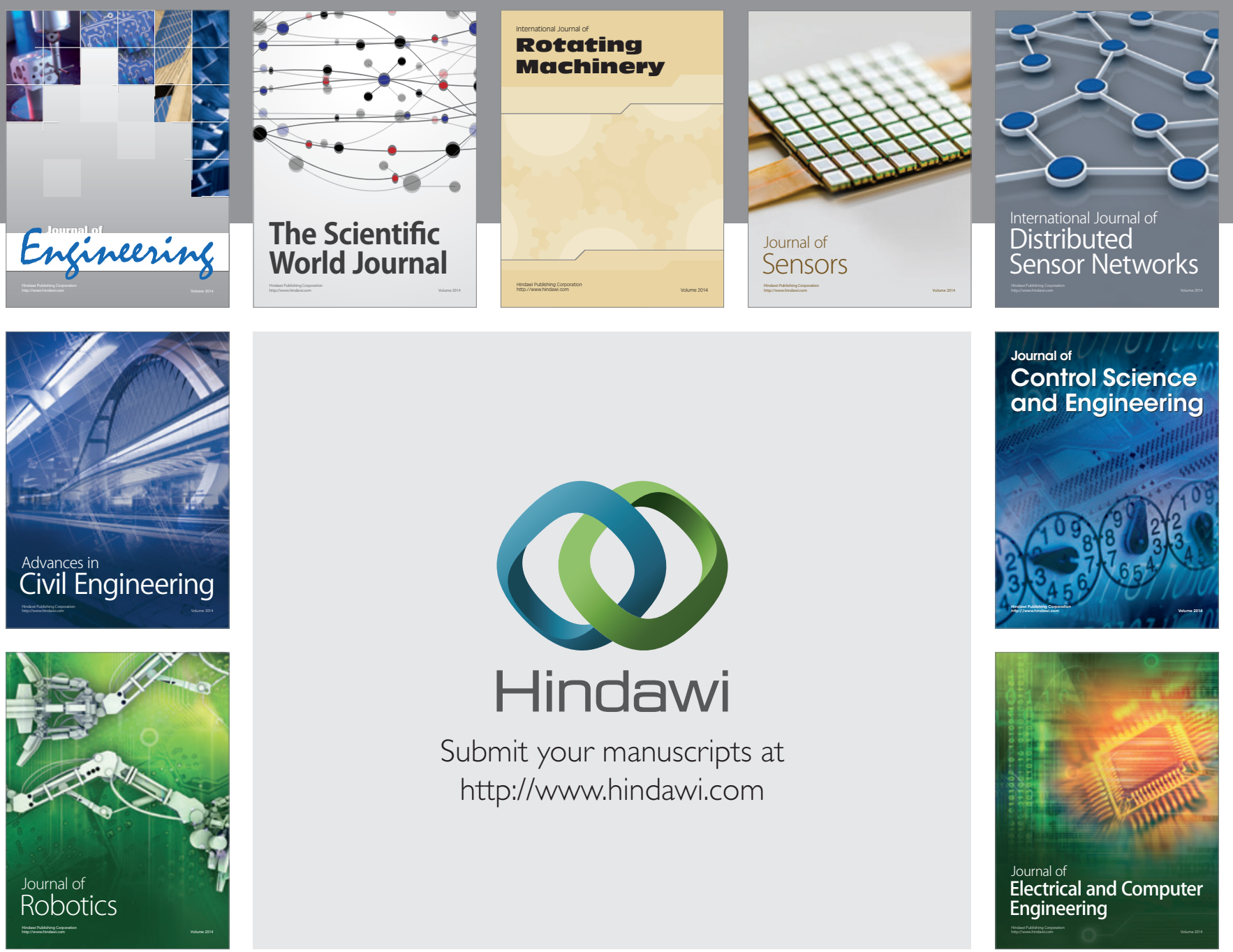

Submit your manuscripts at

http://www.hindawi.com
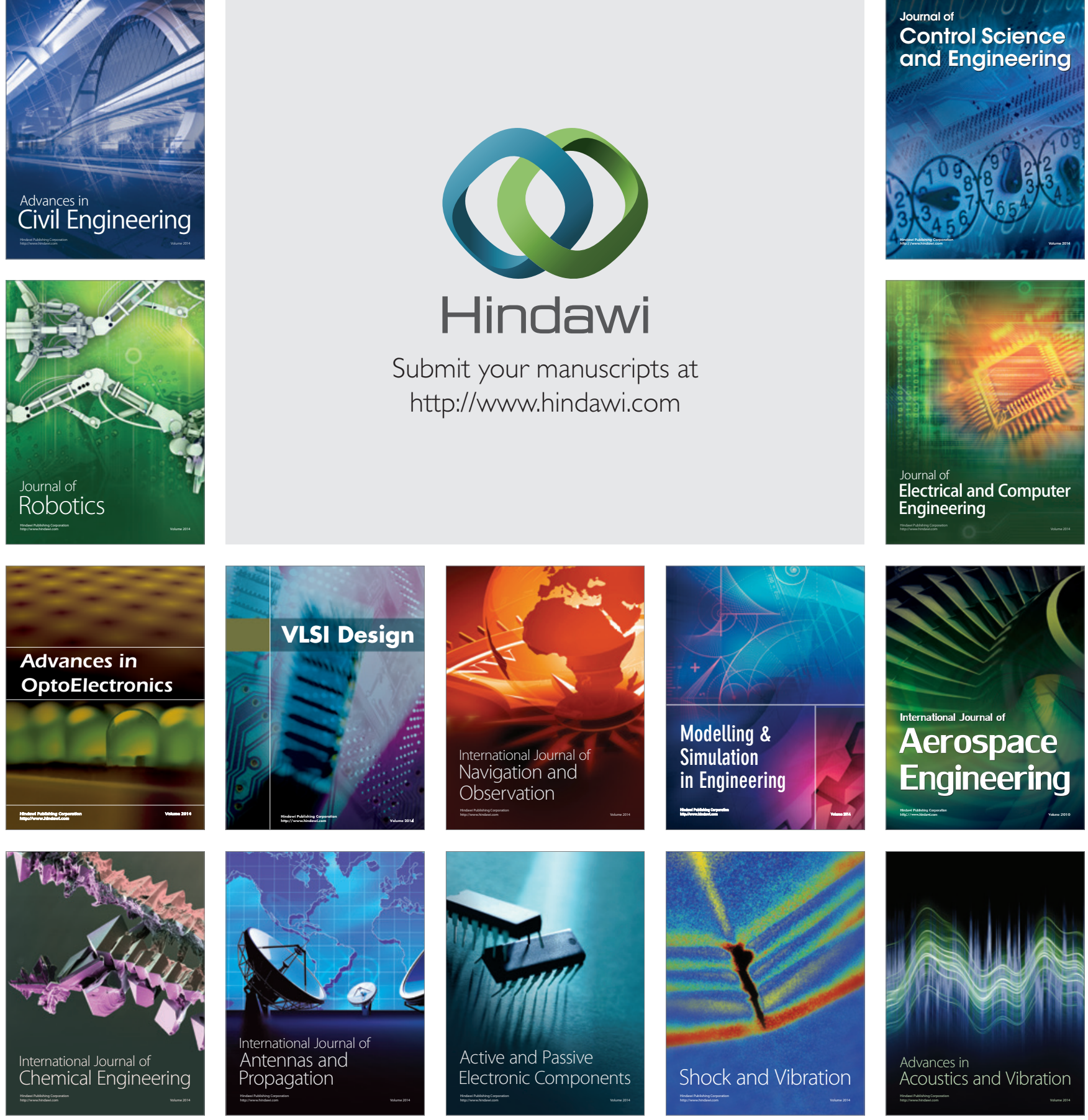